\title{
Metodología para el desarrollo del pensamiento computacional en tiempos de COVID-19 \\ Methodology for the development of Computational Thinking in times of COVID-19
}

\author{
Rafael Herrero-Álvarez ${ }^{1}$, Coromoto León ${ }^{1}$, Gara Miranda ${ }^{1}$, Eduardo Segredo, ${ }^{1}$, Óscar Socas $^{1}$, \\ Laura García ${ }^{2}$, Yolanda Díaz \\ rafael.herrero.13@ull.edu.es, cleon@ull.edu.es,gmiranda@ull.edu.es, esegredo@ull.edu.es, alu0100768152@ull.edu.es, \\ lgarciad@fg.ull.es, ydiaz@fg.ull.es
}

${ }^{1}$ Departamento de Ingeniería Informática y de Sistemas

Universidad de La Laguna

San Cristóbal de La Laguna, España
${ }^{2}$ Cienci@ULL

Fundación General de la Universidad de La Laguna

San Cristóbal de La Laguna, España

\begin{abstract}
Resumen- En este trabajo se describe la metodología seguida con el proyecto 'Piensa@ Computacion@ULLmente' para desarrollar habilidades de pensamiento computacional en estudiantes de $4^{\circ}$ de primaria y $2^{\circ}$ de la Educación Secundaria Obligatoria en tiempos de COVID-19, adaptando intervenciones en el aula y ejercicios a una modalidad sin presencialidad. La propuesta desarrollada se basa en dos vertientes. Por un lado, píldoras educativas en formato vídeo que permiten una realización asíncrona del proyecto, y por otro, intervenciones síncronas con los estudiantes en el aula mediante el uso de software de videoconferencia. Finalmente, se presenta como resultado una comparativa del interés generado en los participantes de este proyecto en anteriores ediciones con presencialidad y con la nueva metodología que se describe.
\end{abstract}

Palabras clave: pensamiento computacional, Educación Primaria, Educación Secundaria, aprendizaje en línea.

Abstract- This paper describes the methodology followed with the 'Piens@Computacion@ULLmente' project to develop Computational Thinking skills considering children of KS2, 4th and KS3, 8th year in times of COVID-19, adapting classroom interventions and exercises to a telematic modality without presence. The proposal developed is based on two strands. On the one hand, educational video pills that allow an asynchronous implementation of the project, and on the other, synchronous interventions with students in the classroom using videoconferencing software. Finally, the results of a comparison studying the interest that this project has generated in the participants in previous editions face-to-face and with the new methodology that is described are presented.

Keywords: Computational Thinking, Primary Education, Secondary Education, e-learning.

\section{INTRODUCCIÓN}

La Informática es un ámbito que cada vez está más presente en nuestro día a día. Concretamente sobre las Tecnologías de las Información y la Comunicación (TIC) en la educación española de los últimos 30 años se aprecia un mayor crecimiento durante la última década de 2010 (Juana Escalona Fernández, Pilar Gómez Martín, \& Isabel Escalona Fernández, 2017). Esto implica que sea necesario promover el conocimiento de los ordenadores y su funcionamiento desde el ámbito educativo preuniversitario. Es por ello por lo que algunos autores muestran la necesidad de realizar un gran cambio en los currículos educativos sobre las Ciencias de la Computación, abordando los diferentes aspectos clave y métodos en los que deben enfocarse, y reflejando que todos los estudiantes deben aprender sobre ellos (Webb et al., 2017). Sin embargo, las Ciencias de la Computación son algo que no siempre despierta interés entre los jóvenes, ya sea por la falta de conocimiento sobre este tema o por percibirlo como algo complejo y fuera del alcance de ellos (Giannakos, Jaccheri, \& Proto, 2013; Hubwieser et al., 2011).

En el caso de España, no existe ninguna estrategia definida para incorporar las Ciencias de la Computación en los currículos educativos (José Manuel Cabrera-Delgado, 2017). De hecho, uno de los problemas principales es que cada comunidad autónoma ha introducido estos contenidos de manera diferente. Por ejemplo, la Comunidad de Madrid ha implantado la asignatura 'Tecnología, Programación y Robótica' en Secundaria, de manera que se enseña a crear programas con diferentes lenguajes de programación, tanto visuales como textuales (Boletín Oficial de la Comunidad de Madrid, 2015). En nuestra comunidad autónoma no se ha incorporado ninguna nueva asignatura, sino que se han redefinido los contenidos de la de 'Tecnología' en Secundaria, añadiendo nuevos bloques como 'Control y robótica', donde es necesario hacer uso de lenguajes de programación (Boletín Oficial de Canarias, 2016).

Existen iniciativas que buscan acercar todo este mundo a los estudios preuniversitarios, como CODE.org con el proyecto de la hora de código (Code.org, 2021c), donde los estudiantes 
realizan una introducción de una hora de duración a las Ciencias de la Computación, o Google con CS First (Google, 2021), con la que se busca facilitar la enseñanza de la programación y hacerla divertida de aprender. Además, también existen algunas con ejercicios desenchufados, es decir, en los que no es necesario el uso de un ordenador o tablet, como CS Unplugged (Computer Science Education Research Group, University of Canterbury, 2021). También existen aplicaciones y herramientas como Scratch (Resnick et al., 2016) con la que es posible programar libremente utilizando un lenguaje de programación visual basado en bloques o Arcade Makecode de Microsoft (Microsoft, 2021) en el que es posible crear un videojuego utilizando bloques o fragmentos de código en Javascript o Python.

En el año 2017 nació el proyecto 'Piens@ Computacion@ULLmente’ (Herrero Álvarez, Segredo, Miranda Valladares, \& Leon, 2019) con el objetivo de acercar las Ciencias de la Computación a los jóvenes mediante el entrenamiento de habilidades del pensamiento computacional en las aulas de Primaria y Secundaria. El pensamiento computacional es un procedimiento por el cual es posible resolver problemas, diseñar sistemas y comprender la conducta humana haciendo uso de conceptos fundamentales de las Ciencias de la Computación (Wing, 2006). Sin embargo, algunos autores también creen que esta habilidad va más allá de las computadoras, destacando tres áreas como los conceptos de programación, prácticas que se desarrollan en la persona según va aprendiendo a programar (reutilizar, mezclar diferentes proyectos, etc.) y perspectivas del mundo que les rodea (cuestionar ideas, establecer contacto con otras personas, etc.) (Brennan, Resnick, \& Media Lab, 2012).

El resto del trabajo está organizado en los siguientes apartados: en la sección 2 se describe el contexto en el que se ha realizado el proyecto. La sección 3 presenta la metodología seguida con las diferentes actividades realizadas. La sección 4 contiene los resultados de este trabajo. Finalmente se presentan las conclusiones además de las líneas de trabajo que se seguirán en el futuro.

\section{CONTEXTO}

En este trabajo se describe la metodología de un proyecto cuyo principal objetivo es el de acercar las Ciencias de la Computación, mediante el entrenamiento de habilidades de pensamiento computacional, en estudios preuniversitarios, de forma que estos estudiantes comprendan lo que son y de esta manera generar interés en ellos, especialmente en las chicas.

Para conseguir este objetivo se propone a los estudiantes que realicen tanto actividades enchufadas como desenchufadas para trabajar conceptos del pensamiento computacional como el reconocimiento de patrones, la abstracción, la descomposición, la lógica o la depuración. Se llevan a cabo cinco sesiones presenciales de 10 horas para el alumnado de $4^{\circ}$ de primaria y de $2^{\circ}$ de la Enseñanza Secundaria Obligatoria (ESO) por un investigador que se desplaza a los centros para impartirlas. Los contenidos se desarrollan bajo dos modalidades de aprendizaje, una guiada, en la que se les da una serie de pautas al alumnado para que resuelva el ejercicio planteado, contando con ayuda si lo necesitase, y otra por descubrimiento, en la que se enseña el uso de una herramienta de tal forma que el estudiante sea capaz de resolver un problema de manera autónoma. Además, estos también realizan una serie de cuestionarios con los que es posible estudiar la percepción que tienen de las Ciencias de la Computación antes y después de las intervenciones o qué modalidad genera mayor interés en ellos.

Debido a la pandemia causada por la COVID-19 fue necesario replantear la metodología que se seguía en ese momento y con la que se ofrecían los contenidos durante el curso escolar 2019/2020, y el que suponía la tercera edición del proyecto, ya que el cierre de los centros educativos obligó a que las sesiones se tuvieran que impartir de manera telemática. Igualmente, durante el curso 2020/2021, debido a las medidas adoptadas para frenar el avance de la pandemia, tampoco se permitía el acceso a las aulas de ninguna persona que no formase parte del centro.

Con estas dos situaciones se plantearon dos soluciones diferentes que permitían darle continuidad al proyecto. Estas fueron:

- Curso 2019/2020: se paralizan los estudios que se llevaban a cabo con los cuestionarios y se generan una serie de materiales audiovisuales con los diferentes contenidos que se trabajaban, resultando en un total de cinco módulos y 19 píldoras de vídeo. Además, se diseñó un aula virtual en el que alojar todo este contenido disponible de manera abierta en Internet.

- Curso 2020/2021: al no poder desplazarse a los centros de manera presencial, se llevan a cabo las cinco sesiones en cada centro de manera telemática y síncrona con los estudiantes que se encuentran en los centros.

\section{DESCRIPCIÓN}

La metodología que se ha seguido para el entrenamiento del pensamiento computacional de manera telemática ha estado centrada en la producción de vídeos y en sesiones de videoconferencias.

\section{A. Píldoras educativas y cuadernillo digital}

En el caso del curso escolar 2019/2020, el 13 de marzo de 2020 se decreta el cierre de los centros, por lo que ante la imposibilidad de continuar el proyecto como se había realizado en las cuatro ediciones anteriores, se realizan 19 píldoras educativas en formato vídeo, disponibles en YouTube (CienciaULL, 2020), como se detallan a continuación:

\section{- Módulo 1. Pensamiento computacional}

- Píldora 1. ¿Qué es el pensamiento computacional? (se explica este concepto).

- Módulo 2. Actividades desenchufadas.

- Píldora 2. Secuencia (se explica que es una secuencia y se realizan las actividades para trabajarlo).

- Píldora 3. Bucle (se explica que es un bucle y se realizan las actividades para trabajarlo).

- Píldora 4. Condicionales (se explica que es un condicional y se realizan las actividades para trabajarlo). 
- Píldora 5. Funciones (se explica que es una función y se realizan las actividades para trabajarlo).

- Píldora 6. Búsqueda (se explica el funcionamiento de un algoritmo de búsqueda binaria).

- Píldora 7. Ordenación (se explican los algoritmos de ordenación, concretamente el de ordenación por pares).

- Módulo 3. Lenguajes de programación visual.

- Píldora 8. Plataforma CODE (se explica el funcionamiento y las características de la plataforma CODE.org).

- Módulo 4. Actividades enchufadas. Nivel 1.

- Píldora 9. Scratch: Fondo, Objetos, Cesta de fruta (se realiza la primera parte de un ejercicio en Scratch para Primaria, como toma de contacto con la plataforma).

- Píldora 10. Scratch: Bloques, Secuencias, Condicionales (se realiza la segunda parte de un ejercicio en Scratch para Primaria, introduciendo nuevos conceptos).

- Píldora 11. Scratch: Variables (se realiza la tercera y última parte de un ejercicio en Scratch para Primaria, introduciendo las variables).

- Módulo 4. Actividades enchufadas. Nivel 2.

- Píldora 12. Scratch: Fondo, Objetos, Globos (se realiza la primera parte de un ejercicio en Scratch para Secundaria, como toma de contacto con la plataforma).

- Píldora 13. Scratch: Bloques, Secuencias (se realiza la segunda parte de un ejercicio en Scratch para Secundaria, introduciendo el concepto de secuencia).

- Píldora 14. Scratch: Bucles, condicionales (se realiza la segunda parte de un ejercicio en Scratch para Secundaria, introduciendo nuevos conceptos).

○ Píldora 15. Scratch: Funciones, Variables (se realiza la tercera parte de un ejercicio en Scratch para Secundaria, introduciendo funciones y variables).

- Píldora 16. Scratch: Ordenación (se realiza la cuarta y última parte de un ejercicio en Scratch para Secundaria, ordenando diferentes objetos según su tamaño).

- Módulo 5. Aplicaciones tangibles.

- Píldora 17. Makey-Makey (se explica el funcionamiento de esta placa y se realiza un ejercicio para probarla).
○ Píldora 18. Mbot (se explica el funcionamiento de este robot y se realiza un ejercicio para probarlo).

- Píldora 19. Roblockly (se explica el funcionamiento de este simulador y se realiza un ejercicio para probarlo).

Con todas estas píldoras se buscaba que el alumnado pudiera seguir, o comenzase, con el desarrollo de las habilidades de pensamiento computacional de una manera autónoma, trabajando todos los conceptos que se trataban de manera presencial, $\mathrm{y}$ con actividades tanto enchufadas como desenchufadas.

Además, también se diseñó y publicó de manera abierta en Internet un curso, bajo el servicio de Open Course Ware (OCW) (Universidad de La Laguna, 2021), que contiene y ordena todas estas píldoras según los módulos descritos, además de materiales adicionales para que cualquier persona pueda realizarlo sin necesitar de un docente.

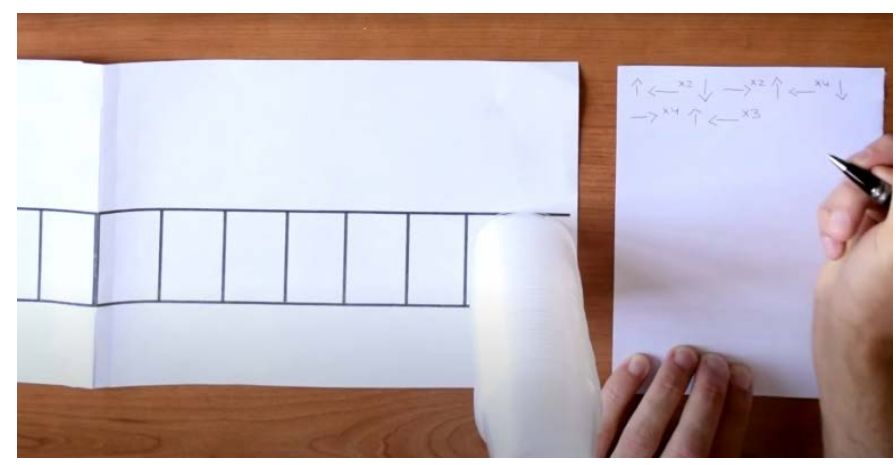

Figura 1: captura de la píldora 3 sobre bucles en la que se realiza un castillo de vasos utilizando únicamente flechas como instrucciones

\section{B. Sesiones telemáticas síncronas}

En el curso escolar 2020/2021 se llevaron a cabo cinco sesiones de dos horas, al igual que se venía realizando en las anteriores ediciones de Piens@,Computacion@ULLmente. Sin embargo, en esta ocasión los investigadores no se desplazaron a los centros educativos, sino que se llevaron a cabo mediante el uso de la plataforma Google Meet. Debido a esto, ahora el investigador se encontraba en un plató y establecía conexión con el aula, las cuales contaban con un proyector y una cámara con micrófono, por lo que era posible una comunicación bidireccional, como se puede apreciar en la Figura 2.

Las sesiones se organizaron teniendo en cuenta los conceptos de programación de secuencias, condicionales, bucles, variables y funciones. Para cada una de ellas se realizaban dos actividades, una desenchufada y otra enchufada. En el caso de las sesiones en las que fuese necesario algún tipo de material, este era entregado por la organización del proyecto previamente.

Las actividades realizadas en Primaria han sido:

- Sesión 1. Secuencias: recorriendo el laberinto (resolver un laberinto utilizando flechas como instrucciones, desenchufada) y Curso 2 de CODE.org (Code.org, 2021a) (apartados del 1 al 4, enchufada). 
- Sesión 2. Condicionales: árbol de decisión y ordenación por pares (adivinar un número preguntando si es mayor o igual o no y dibujar su árbol de decisión, así como ordenar una serie de números de manera ascendente utilizando un algoritmo de ordenación por pares, desenchufada) y Curso 2 de CODE.org (Code.org, 2021a) (apartados del 13 al 16, enchufada).

- Sesión 3. Bucles: programando un dibujo (siguiendo unas instrucciones de flechas y de pintar, moverse por un tablero y pintar en las casillas señaladas formando un dibujo, desenchufada) y figuras en Scratch (programar un objeto para que dibuje las figuras de cuadrado, triangulo y círculo, enchufada).

- Sesión 4. Variables: sobres variables (pintar un monstruo teniendo 6 sobres donde cada sobre representa una variable y contiene características como número de piernas, forma de cabeza, etc., desenchufada) y explotando globos en Scratch (programar dos objetos para que se puedan pinchar globos que aparecen en pantalla con un lápiz, enchufada).

- Sesión 5. Funciones: mi propio mando a distancia (dibujar un mando a distancia con varios botones en un folio, cada persona con una tarjeta con uno de los botones que contiene una frase diferente. Al pulsar en el mando a distancia se forma una canción, adivinanza, etc., desenchufada) y PONG en Scratch (programar variante del juego PONG, enchufada).

Las actividades realizadas en Secundaria han sido:

- Sesión 1. Secuencias: castillos de vasos (programar a un robot con flechas para que monte diferentes castillos partiendo de una pila de vasos, desenchufada) y Curso 2 de CODE.org (Code.org, 2021a) (apartados del 1 al 4, enchufada).

- Sesión 2. Condicionales: ordenación por pares y algoritmos de ordenación (ordenar una serie de números de manera ascendente utilizando un algoritmo de ordenación por pares y mostrar funcionamiento de los algoritmos de ordenación de burbuja, inserción y radix, desenchufadas) y Curso D de CODE.org (Code.org, 2021b) (apartados del 10 al 13 , enchufada).

- Sesión 3. Bucles: recorriendo el laberinto y castillos de vasos (resolver un laberinto utilizando flechas con bucles como instrucciones y misma actividad de la sesión 1, pero utilizando bucles, desenchufadas) y Curso 2 de CODE.org (apartados del 5 al 8, enchufada).

- Sesión 4. Variables: laberinto con variables (recorrer un laberinto con diferentes operaciones matemáticas en las que se van actualizando los valores de las variables, desenchufada) y PONG en Scratch (programar variante del juego PONG, enchufada).
- Sesión 5. Funciones: héroe o villano (juego en el que tres participantes tienen una función diferente: un héroe protege una ciudad de los ataques de un villano mientras un vigilante controla los daños causados, de tal forma que no se supere una cantidad determinada de ataque siguiendo unas condiciones establecidas previamente, desenchufada) y atrapar el ratón en Scratch (programar un gato y un ratón para hacer que el primero atrape al segundo que se mueve libremente por la pantalla, utilizando las flechas de dirección del teclado, enchufada).

Debido a que los investigadores no podían desplazarse a los centros, se llevó a cabo un curso de formación del profesorado previo al comienzo de las sesiones, ya que este se convertiría en un guía en el aula física. También, se diseñaron plantillas con descripciones detalladas de cada una de las actividades, con las que era posible seguir las instrucciones de las sesiones y de los ejercicios, así como consultar que material era necesario para cada estudiante para realizar las actividades, por ejemplo, los vasos de plástico para las sesiones de secuencias o bucles, o los sobres para las de variables. Junto a estas guías también se incluían las soluciones y una versión de material para imprimir, de forma que los estudiantes no tuviesen acceso a las soluciones.

Para facilitar al profesorado el acceso a toda la información se implementaron diferentes aulas virtuales, una para cada centro participante, en la que se alojaron, de forma esquematiza y centralizada, las plantillas de cada una de las actividades, dos por sesión, una para la actividad desenchufada y otra para la enchufada, además del material para imprimir y de un cronograma en el que se especificaban las actividades que se iban a hacer cada día.

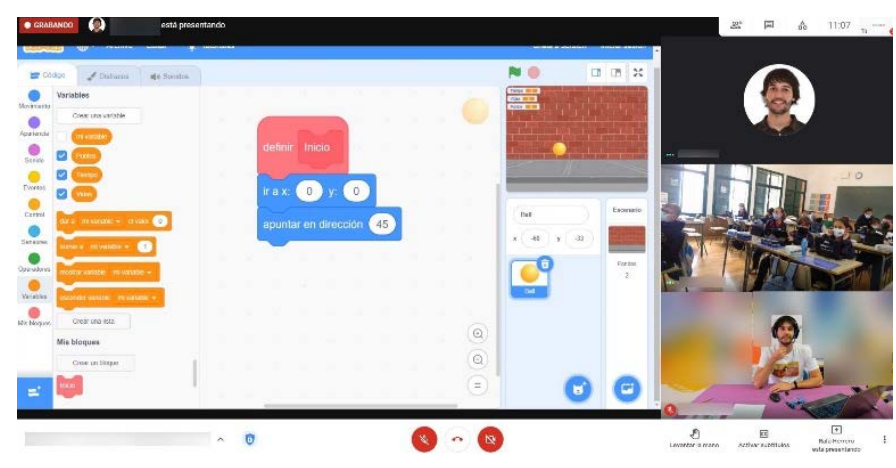

Figura 2: captura de una de las sesiones telemáticas sobre funciones con los centros

\section{Resultados}

Para evaluar el nivel de impacto de la metodología de las actividades descrita en los estudiantes, se realizó una comparación entre los resultados a la pregunta " $¿$ Te han gustado las actividades de pensamiento computacional que hemos realizado?", la cual tenían que responder todos los estudiantes al finalizar la quinta y última sesión del proyecto mediante un Google Form. 
Tabla 1: descripción cuantitativa de la muestra

\begin{tabular}{|c|c|c|c|c|c|c|c|}
\hline \multicolumn{3}{|c|}{ PRESENCIAL } & \multicolumn{4}{c|}{ TELEMÁTICO } \\
\hline \multicolumn{2}{|c|}{ PRIMARIA } & SECUNDARIA & PRIMARIA & \multicolumn{2}{c|}{ SECUNDARIA } \\
\hline Chica & Chico & Chica & Chico & Chica & Chico & Chica & Chico \\
\hline 207 & 203 & 208 & 222 & 40 & 33 & 85 & 101 \\
\hline
\end{tabular}

Cabe mencionar que, de la tercera edición, la del curso escolar 2019/2020, los resultados que se muestran son todos en modalidad presencial, puesto que en el momento en el que se decretó el cierre de los centros escolares se cambió a la metodología de las píldoras de vídeos, las cuales no tenían asociado ningún cuestionario ni ningún tipo de recogida de datos. En la Tabla 1 se recogen el total de alumnos que realizaron el cuestionario, separando según haya realizado actividades presenciales o de manera telemática, el nivel educativo y el género.

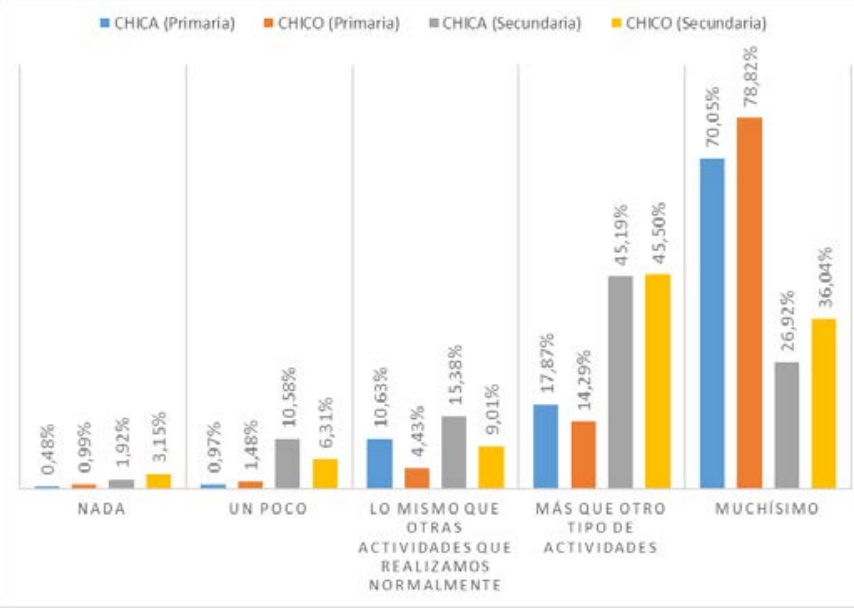

Figura 3: resultados de la pregunta "¿Te han gustado las actividades de pensamiento computacional que hemos realizado?" para la modalidad presencial

Como se aprecia en la Figura 3, estas actividades generan bastante interés en los estudiantes cuando se realizan de manera presencial. Existe una gran diferencia entre los estudiantes de Primaria, donde a un 74,39\% les han gustado muchísimo, frente a los de Secundaria, donde solo ha sido a un $31,63 \%$. Sin embargo, a un $45,35 \%$ de los estudiantes de Secundaria les ha gustado más que otro tipo de actividades, por lo que la respuesta es mayormente positiva. En cuanto a que no les gusta nada o un poco, solo un 1,95\% y 10,93\% de los estudiantes de Primaria y Secundaria respectivamente, opinan así.

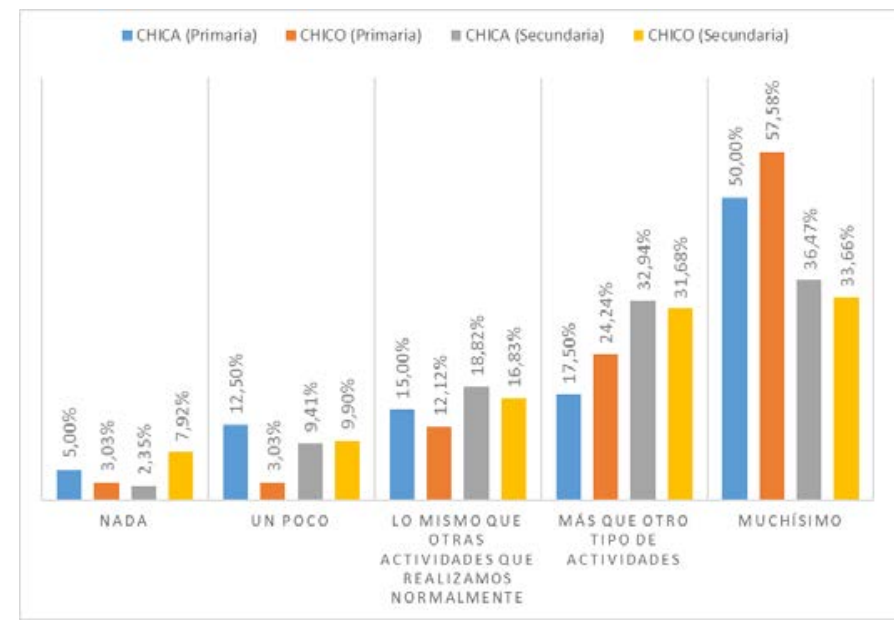

Figura 4: resultados de la pregunta "¿Te han gustado las actividades de pensamiento computacional que hemos realizado?" para la modalidad telemática

En la Figura 4 se recogen las valoraciones para las actividades que se han realizado de manera telemática. La respuesta es positiva mayoritariamente, ya que a un $73,97 \%$ de los estudiantes de Primaria y a un 67,20\% de los de Secundaria, les gusta más que otro tipo de actividades o muchísimo. Por otro lado, se observa que a un $12,32 \%$ del alumnado de Primaria y a un $15,05 \%$ de los de Secundaria, no les ha gustado nada o un poco.

Estas gráficas reflejan que los estudiantes prefieren las actividades presenciales sobre las telemáticas, especialmente en Primaria, ya que a un $74,39 \%$ de los estudiantes les ha gustado muchísimo de manera presencial, frente a un $53,42 \%$ en el caso de la modalidad telemática. También resulta llamativo el aumento del alumnado al que no le ha gustado nada o solamente un poco el realizar las actividades de manera telemática, ya que en el caso de la presencialidad el porcentaje era de un $6,55 \%$ y este aumenta hasta un $14,29 \%$ en la modalidad en línea.

\section{CONCLUSIONES}

Este trabajo contiene la metodología seguida en el proyecto Piens@Computacion@ULLmente debido a la pandemia causada por la COVID-19, ya que hasta el momento este se realizaba de manera presencial y obligó a una adaptación telemática.

A partir del curso escolar 2020/2021 se realizaban las sesiones desde un plató utilizando Google Meet, conectando con las aulas, y en las cuales el profesorado presente tenía que comprobar que el alumnado seguía correctamente las instrucciones dadas.

Previo al comienzo del proyecto, el profesorado recibía, de manera optativa, un curso con el objetivo de que adquiriesen habilidades de pensamiento computacional y se implicasen en el desarrollo de las sesiones en el aula. No todos los docentes participaron en este curso, por lo que esta falta de implicación podría ser una de las razones de las diferencias observadas entre la metodología presencial y la telemática.

En el caso de la modalidad presencial, se realizaban también actividades con el robot mBot (Makeblock, 2021) o la placa Makey Makey (JoyLabz, 2021), por lo que los estudiantes tenían elementos físicos que programar, y debido a la situación 
generada por la COVID-19 no se realizaron. Esto podría ser una de las razones por las que la modalidad telemática no ha generado la misma respuesta que la presencial.

En trabajos futuros se estudiará la diferencia a la hora de entrenar habilidades de pensamiento computacional según se hayan realizado las actividades de manera presencial o telemática, de forma que sea posible determinar cuál de ellas resulta mejor para los estudiantes preuniversitarios.

\section{AGRADECIMIENTOS}

Este proyecto ha sido financiado por una subvención directa del Cabildo de Tenerife y se enmarca en la actividad de "Piens@Computacion@ULLmente (A17120413). Programa educativo para el fomento del pensamiento computacional a través de la realización de actividades que permitan su desarrollo y su inclusión en el currículo".

\section{REFERENCIAS}

Decreto 83/2016, de 4 de julio, por el que se establece el currículo de la Educación Secundaria Obligatoria y el Bachillerato en la Comunidad Autónoma de Canarias. (2016).

Decreto 48/2015, de 14 de mayo, del Consejo de Gobierno, por el que se establece para la Comunidad de Madrid el currículo de la Educación Secundaria Obligatoria, (2015).

Brennan, K., Resnick, M., \& Media Lab, M.New frameworks for studying and assessing the development of Computational Thinking.

CienciaULL, U. (2020). Lista piens@ Computacion@ULLmente.r Retrieved from https://www.youtube.com/playlist?list=PLyCiNKvX7K OFHb3OIb0t_VLoos5bE_xBd

Code.org. (2021a). Curso 2 - Code.org. Retrieved from https://studio.code.org/s/course2

Code.org. (2021b). Curso D - Code.org. Retrieved from https://studio.code.org/s/coursed-2017

Code.org. (2021c). Hour of code. Retrieved from https://hourofcode.com/es

Computer Science Education Research Group, University of Canterbury. (2021). CS Unplugged. Retrieved from https://csunplugged.org

Giannakos, M. N., Jaccheri, L., \& Proto, R.Teaching computer science to young children through creativity: Lessons learned from the case of Norway. Paper presented at the 103-111.

Google. (2021). Enseña informática y programación a niños CS First. Retrieved from https://csfirst.withgoogle.com/

Herrero Álvarez, R., Segredo, E., Miranda Valladares, G., \& Leon, C. (2019). (2019). El proyecto Piens@ Computacion@LLmente. Paper presented at the 573-578. doi:10.26754/CINAIC.2019.0117

Hubwieser, P., Armoni, M., Brinda, T., Dagiene, V., Diethelm, I., Giannakos, M., . . . Schubert, S. (2011). Computer science/informatics in secondary education. Proceedings of the 16th Annual Conference Reports on Innovation and Technology in Computer Science Education - Working Group Reports, , 19-38. doi:10.1145/2078856.2078859

José Manuel Cabrera-Delgado. (2017). Las Ciencias de la Computación en el currículo educativo. Avances en Supervisión Educativa, (27) doi:10.23824/ase.v0i27.584

JoyLabz. (2021). Makey makey. Retrieved from https://makeymakey.com/

Juana Escalona Fernández, Pilar Gómez Martín, \& Isabel Escalona Fernández. (2017). Las TIC en la educación española a través de las publicaciones periódicas: Un análisis bibliométrico. Pixel-Bit : Revista De Medios Y Educación, (51) doi:10.12795/pixelbit.2017.i51.02

Makeblock. (2021). mBot. Retrieved from https://www.makeblock.com/mbot/

Microsoft. (2021). Microsoft MakeCode arcade. Retrieved from https://arcade.makecode.com

Resnick, M., Maloney, J., Monroy-Hernández, A., Rusk, N., Eastmond, E., Brennan, K., . . . Kafai, Y. (2016). Scratch: Programming for all. Communications of the ACM, 52(11), 60-67. doi:10.1145/1592761.1592779

Universidad de La Laguna. (2021). Cuadernillos digitales de pensamiento computacional. Retrieved from https://campusvirtual.ull.es/ocw/course/view.php?id=153

Webb, M., Davis, N., Bell, T., Katz, Y., Reynolds, N., Chambers, D., \& Sysło, M. (2017). Computer science in K-12 school curricula of the 2lst century: Why, what and when? Education and Information Technologies, 22(2), 445-468. doi:10.1007/s10639-016-9493-x

Wing, J. M. (2006). Computational Thinking. Communications of the Acm, , 33-35. 